\title{
SUMS OF DISTANCES BETWEEN POINTS ON A SPHERE
}

\author{
KENNETH B. STOLARSKY 1
}

\begin{abstract}
An upper bound for the sum of the $\lambda$ th powers of all distances determined by $N$ points on a unit sphere is given for $0 \leqq \lambda \leqq 1$.
\end{abstract}

Let $p_{1}, \cdots, p_{N}$ be points on the unit sphere $U^{m}$ of $E^{\prime \prime}$, the $m$-dimensional Euclidean space. Let

$$
S=S(N, m, \lambda)=\max \sum_{i<j}\left|p_{i}-p_{j}\right|^{\lambda}
$$

where the maximum is taken over all possible $p_{1}, \cdots, p_{x}$. The function $S$ has been studied in [1]-[5]. Let $\sigma\left(U^{m}\right)$ denote the surface area of $U^{m}$. Björck has shown in [3] that $S<c(m, \lambda) N^{2}$ where $c(m, \lambda)=$ $\frac{1}{2} \sigma\left(U^{m}\right)^{-1} \int\left|p_{0}-p\right|^{\lambda} d \sigma(p)$; here $\int \cdots d \sigma(p)$ denotes a surface integral over $U^{m}$ and $p_{0}$ is an arbitrary point on $U^{m}$. For example, $c(3,1)=\frac{2}{3}$. In [1] Alexander used Haar integrals to prove

$$
S(N, 3,1)<\frac{2}{3} N^{2}-\frac{1}{2}
$$

By the same technique we will show (see comment after Theorem 2) that for $0 \leqq \lambda \leqq 1$,

$$
S(N, m, \lambda) \leqq c(m, \lambda) N^{2}-\frac{1}{2} c(m, \lambda)
$$

is true at least "half of the time"; more precisely, for any positive integer $n$ the inequality (2) is true either for $N=n$ or for $N=n+1$. Our proof is based on a new generalization of the triangle inequality, Theorem 1, and an imbedding theorem of I. J. Schoenberg [6] or [7, p. 527]. Note that when $m=3$ and $\lambda=1$ our result is weaker than Alexander's.

LEMMA 1. Let $u \geqq v+2 \geqq 3$ and let $H$ denote the hexagonal region $0 \leqq x \leqq u, 0 \leqq y \leqq v, 1 \leqq x+y \leqq u+v-1$. Then for $(x, y)$ in $H$ we have

$$
g(x, y)=\frac{x(u-x)+y(v-y)}{x(v-y)+y(u-x)} \leqq \frac{u-1}{v} .
$$

Received by the editors December 29, 1971.

AMS 1970 subject classifications. Primary 52A25, 52A40.

${ }^{1}$ Research supported in part by the National Science Foundation under contract GP-12363. 
Proof. Let $s$ denote the substitution which takes $x$ and $y$ to $u-x$ and $v-y$ respectively. The function $g(x, y)$ is invariant under $s$, so it suffices to prove the inequality for the three critical points $\left(\frac{1}{4}(u+v), \frac{1}{4}(u+v)\right)$, $\left(\frac{1}{2}(u+v), \frac{1}{2}(u+v)\right),\left(\frac{1}{2} u, \frac{1}{2} v\right)$, and the three boundary lines $x+y=1, y=0$ and $x=u$. The straightforward details are omitted.

Now let $S_{1}$ be a finite set of $u$ points $p_{1}, \cdots, p_{u}$ in $E^{m}$ and similarly let $S_{2}$ be a set of $v$ such points $q_{1}, \cdots, q_{v}$. Let $\sum_{1}=\Sigma_{i<j}\left|p_{i}-p_{j}\right|, \sum_{2}=$ $\sum_{i<j}\left|q_{i}-q_{j}\right|$, and $\sum_{12}=\sum_{i, j}\left|p_{i}-q_{j}\right|$.

Theorem 1. Let $u \geqq v$. Define

Then

$$
\begin{aligned}
f(u, v) & =1, & & u=v, \\
& =(u-1) / v, & & u>v .
\end{aligned}
$$

$$
\sum_{1}+\sum_{2} \leqq f(u, v) \sum_{12}
$$

Moreover the constant $f(u, v)$ is best possible.

Proof. For any two points $p_{1}, p_{2} \in E^{m}$,

$$
\left|p_{1}-p_{2}\right|=a_{m} \int\left|p_{1} \cdot p-p_{2} \cdot p\right| d \sigma(p)
$$

where $a_{m}$ depends only on the dimension $m$. Hence it suffices to prove (3) in the case where the $p_{i}$ and $q_{j}$ are real numbers. Let $J$ be any interval on the real axis which does not contain any point $p_{i}$ or $q_{j}$. Let $x$ be the number of $p_{i}$ to the left of $J$ and $y$ the number of $q_{j}$ to the left of $J$. It suffices to show $L \leqq f(u, v) R$ where $L$ is the number of times $J$ is counted on the left of (3) and $R$ is the number of times it is counted on the right, i.e. that

$$
x(u-x)+y(v-y) \leqq f(u, v)\{x(v-y)+y(u-x)\} .
$$

For $v \leqq u \leqq v+1$ this follows from $(x-y)(u-v) \leqq(x-y)^{2}$ and for $v+2 \leqq u$ it follows from Lemma 1. Equality holds in (3) if (not only if) $p_{2}=p_{3}=$ $\cdots=p_{u}=q_{1}=\cdots=q_{v}$ and $p_{1}$ is arbitrary.

THEOREM 2. For $0 \leqq \lambda \leqq 1$,

$$
S(u, m, \lambda)+S(v, m, \lambda) \leqq 2 c(m, \lambda) u v f(u, v)
$$

Proof. Fix $\lambda$. Given any $t$ points $p_{1}, \cdots, p_{t}$ in $E^{m}$, we can find an $m^{\prime} \geqq m$ and $t$ points $p_{1}^{\prime}, \cdots, p_{t}^{\prime}$ in $E^{m^{\prime}}$ such that $\left|p_{i}^{\prime}-p_{j}^{\prime}\right|=\left|p_{i}-p_{j}\right|^{\lambda}$ for $1 \leqq i, j \leqq t$. This result is due to I. J. Schoenberg [7, p. 527]. In the following $\tau, \tau_{1}, \tau_{2}$ shall denote elements of the special orthogonal group $S O(m)$ acting on $U^{m}$. The symbol $f \cdots d \tau$ shall denote a Haar integral over that group; we normalize the measure so that $f_{S O(m)} d \tau=1$. Choose $p_{1}, \cdots, p_{u}$ 
and $q_{1}, \cdots, q_{v}$ on $U^{m}$ so that

$$
S(u, m, \lambda)=\sum_{i<j}\left|p_{i}-p_{j}\right|^{\lambda} \text { and } S(v, m, \lambda)=\sum_{i<j}\left|q_{i}-q_{j}\right|^{\lambda}
$$

Define

\section{Clearly}

$$
I=f \sum_{i, j}\left|p_{i}-\tau_{1} q_{j}\right|^{\lambda} d \tau_{1}
$$

(6) $I=u v f\left|p-\tau_{1} \cdot p\right|^{\lambda} d \tau_{1}=u v \sigma\left(U^{m}\right)^{-1} \int\left|p_{0}-p\right|^{\lambda} d \sigma(p)=2 c(m, \lambda) u v$

On the other hand, for any $p_{0}$ on $U^{m^{\prime}}$,

$$
\left|p_{i}-\tau \cdot q_{j}\right|^{\lambda}=\left|p_{i}^{\prime}-\left(\tau_{1} q_{j}\right)^{\prime}\right|=b_{m^{\prime}} f\left|\left(p_{i}^{\prime}-\left(\tau_{1} \cdot q_{j}\right)^{\prime}\right) \cdot \tau_{2} p_{0}\right| d \tau_{2}
$$

where $b_{m}$ depends only on the dimension $m^{\prime}$. Hence by Theorem 1 ,

$$
\begin{aligned}
& I=\int f b_{m^{\prime}} \sum_{i, j}\left|p_{i}^{\prime} \cdot \tau_{2} p_{0}-\left(\tau_{1} q_{j}\right)^{\prime} \cdot \tau_{2} p_{0}\right| d \tau_{2} d \tau_{1} \\
& \geqq f^{-1} f f\left(\sum_{i<j} b_{m^{\prime}}\left|\left(p_{i}^{\prime}-p_{j}^{\prime}\right) \cdot \tau_{2} p_{0}\right|\right. \\
& \left.\quad+\sum_{i<j} b_{m^{\prime}}\left|\left\{\left(\tau_{1} q_{i}\right)^{\prime}-\left(\tau_{1} q_{j}\right)^{\prime}\right\} \cdot \tau_{2} p_{0}\right|\right) d \tau_{2} d \tau_{1} \\
& =f^{-1} f\left(\sum_{i<j}\left|p_{i}-p_{j}\right|^{\lambda}+\sum_{i<j}\left|q_{i}-q_{j}\right|^{\lambda}\right) d \tau_{1} .
\end{aligned}
$$

The result follows from (5), (6), and (7).

To obtain (2) set $v=u-1$ in (4) and note that $c u^{2}-\frac{1}{2} c+c(u-1)^{2}-$ $\frac{1}{2} c=2 c u(u-1)$.

\section{REFERENCES}

1. R. Alexander, On the set of distances determined by $n$ points on the 2-sphere (in preparation).

2. R. Alexander and K. B. Stolarsky, Some extremal problems of distance geometry (in preparation).

3. G. Björck, Distributions of positive mass which maximize a certain generalized energy integral, Ark. Mat. 3 (1955), 255-269. MR 17. 1198.

4. L. Fejes Tóth, On the sum of distances determined by a pointset, Acta Math. Acad. Sci. Hungar. 7 (1956), 397-401. MR 21 \#5937.

5. G. Pólya and G. Szegö, Über den transfiniten Durchmesser (Kapazitätskonstante) von ebenen und räumlichen Punktmengen, J. Reine Angew. Math. 165 (1931), 4-49.

6. I. J. Schoenberg, On certain metric spaces arising from Euclidean spaces by change of metric and their imbedding in Hilbert space, Ann. of Math. 38 (1937), 787-793.

7. - Metric spaces and positive definite functions, Trans. Amer. Math. Soc. 44 (1938), 522-536.

Department of Mathematics, University of Illinois, Urbana, Illinois 61801 